\title{
Germanica
}

\section{Lieux étrangers et lieux familiers dans In fremden Gärten de Peter Stamm}

Fremde und vertraute Orte in Peter Stamms In fremden Gärten

Joëlle Stoupy

\section{OpenEdition}

\section{Journals}

Édition électronique

URL : http://journals.openedition.org/germanica/335

DOI : 10.4000/germanica.335

ISSN : 2107-0784

\section{Éditeur}

Université de Lille

\section{Édition imprimée}

Date de publication : 1 décembre 2006

Pagination : 149-161

ISBN : 2-913857-18-3

ISSN : 0984-2632

\section{Référence électronique}

Joëlle Stoupy, «Lieux étrangers et lieux familiers dans In fremden Gärten de Peter Stamm », Germanica

[En ligne], 39 | 2006, mis en ligne le 19 février 2010, consulté le 06 octobre 2020. URL : http:// journals.openedition.org/germanica/335; DOI : https://doi.org/10.4000/germanica.335

Ce document a été généré automatiquement le 6 octobre 2020.

(C) Tous droits réservés 


\title{
Lieux étrangers et lieux familiers dans In fremden Gärten de Peter Stamm
}

\author{
Fremde und vertraute Orte in Peter Stamms In fremden Gärten
}

Joëlle Stoupy

1 Né à Münsterlingen (Suisse) en 1963, Peter Stamm a attiré le regard du grand public en 1998 avec la parution de son roman Agnes qui a notamment reçu le prix de Rauris en Autriche. Vient alors avec Blitzeis (Verglas) en 1999 un recueil de neuf récits, deux ans plus tard, un deuxième roman, Ungefähre Landschaft (Paysages aléatoires) et plus récemment, en 2003, un nouveau recueil de récits dont le titre, In fremden Gärten (D'Étranges jardins), est inspiré d'une phrase des Années d'apprentissage de Wilhelm Meister de Goethe.

2 Comme l'auteur le remarque lui-même, le désir de devenir écrivain ne remonte pas à son enfance, à une époque où être ingénieur en construction navale le tentait bien davantage. S'il avait imaginé le long parcours qui, de ses débuts, l'a mené à la publication de son roman Agnes, il aurait, confie-t-il ${ }^{1}$, peut-être abandonné le projet d'écrire - bien que ce soit une des occupations qui jamais ne provoque chez lui l'ennui. Encore aujourd'hui, il reste perplexe sur le courage qu'il a trouvé, en automne 1990, de suspendre ses études de psychologie pour se consacrer exclusivement à l'écriture. Un fait extérieur l'a aidé à percer. En 1998, la Suisse a été invité d'honneur au Salon du livre de Francfort-sur-le-Main, ce qui lui donna l'opportunité de trouver une maison d'édition (Arche Verlag). Aujourd'hui Peter Stamm vit et travaille à Zurich et Winterthur². Il est écrivain et journaliste notamment pour le Neue Zürcher Zeitung et le Tages-Anzeiger.

3 Si Dürrenmatt fait partie des premiers auteurs qu'il a lus de façon systématique ${ }^{3}$, Peter Stamm pense que le caractère national est de plus en plus secondaire dans la littérature et il avoue se sentir plus proche de certains auteurs allemands que de certains collègues suisses. Il doit à de nombreuses littératures sa formation et dit être influencé par les littératures américaine, allemande, française, italienne, norvégienne, russe et anglaise. 
Pour ajouter : "un peu aussi par la littérature suisse $»^{4}$. Lorsque l'on compare le style minimaliste de Peter Stamm, il n'est pas rare que l'on fasse appel à des auteurs d'OutreAtlantique, tels que Ernest Hemingway ou Raymond Carver. Après la mort des grands auteurs suisses, Max Frisch et Friedrich Dürrenmatt, Peter Stamm fait partie de la jeune génération suisse qui commence à s'établir, sans insister toutefois de façon outrancière sur son héritage suisse.

Dans son dernier recueil de récits, In fremden Gärten, seuls quatre textes parmi onze se déroulent en Suisse ; les autres ont pour coulisse New York, la Lettonie, l'Angleterre, l'Italie, l'Irlande et le Portugal. On a appelé les personnages de Stamm des nomades ${ }^{5}$ parcourant le monde plus ou moins volontairement. L'auteur a lui-même derrière lui une expérience de la vie à l'étranger pour avoir fait de longs séjours aux États-Unis, en France, en Scandinavie, en Allemagne et en Angleterre. Son engouement pour l'étranger a commencé, dit-il, après son premier séjour à Paris, une ville qu'il a haïe les premiers mois mais qu'il a plus tard quittée avec peine. C'est notamment la rupture avec le quotidien qui l'attire à l'étranger et l'opportunité de connaître des modes de vie variés :

Ma nationalité ne m'a jamais paru particulièrement importante. J'ai toujours essayé de m'adapter au pays dans lequel je me trouvais. Autrement, j'aurais tout aussi bien pu rester en Suisse ${ }^{6}$.

Dans le récit qui ouvre le recueil In fremden Gärten et qui de toute évidence se déroule en Suisse, Der Besuch, le goût du voyage s'éveille dans le personnage principal, Regina, tout à la fin, avec l'apparition d'un jeune Australien. L'essentiel du texte repose sur l'appréhension qu'a cette femme de 75 ans de la maison familiale. Regina se voit dans l'obligation de constater que ce lieu familier lui est devenu étranger depuis qu'elle y vit seule et que ses enfants l'ont déserté pour n'y revenir, sans grand enthousiasme, que lors de fêtes familiales. Peter Stamm ne fait qu'esquisser le passé de cette septuagénaire et manie habilement les non-dits. En mettant cependant en avant le sentiment de solitude et de vide qui suit le départ des enfants et le décès de son mari, il présente un bilan d'une vie centrée autour de l'éducation des enfants et de la vie familiale, une vie qui s'étiole sans leur présence. Difficile de dire avec précision quelle est la relation entre le personnage principal, Regina, et ses trois enfants. Ils sont certes attachés à leur mère et présents dans les moments de crise, mais la maison qui les a vu grandir leur est devenue étrangère ; y rester trop longtemps semble même éveiller en eux un malaise. Ainsi ils évitent d'y passer la nuit et préfèrent ne pas garder la clé, démontrant ainsi leur indépendance. C'est à ces petits gestes anodins que Regina ressent leur éloignement. Lors de visites occasionnelles, ils se comportent dans leur ancienne demeure comme des visiteurs dans un musée.

Rien de semblable lors de la venue de l'ami australien de la petite-fille de Regina à la fin du récit. Subitement, la maison qui va abriter pour une nuit l'amour de ces jeunes gens reprend vie, l'espace semble avoir un sens, l'impression de vide et d'étrangeté être anéantie. "Depuis longtemps elle [Regina] avait à nouveau l'impression que la maison était pleine ${ }^{7} »$. L'ami australien, étranger et maîtrisant à peine la langue du pays, est avec Martina, la petite-fille de Regina, le seul à trouver cette grande maison familière, parce qu'elle leur offre l'opportunité d'être ensemble pour une nuit. Pour montrer son 
sentiment de bien-être, il enlève ses chaussures dès qu'il entre, comme il le ferait chez lui ; il éprouve aussi le besoin de montrer à Regina, sur un ordinateur de poche, sa maison en Australie. Le récit se termine sur la détermination de Regina d'entreprendre un voyage qu'elle veut adapté à son âge. Ce ne sera plus ni l'Australie, ni l'Espagne, comprend-elle sans amertume. En voyant sa petite-fille pleine de force et de confiance dans l'avenir, elle semble alors accepter avec plus de sérénité les années qui ont passé et qui lui ont rendu étranger ce qui lui était si familier.

8 Le lieu familier d'Henry, le protagoniste du deuxième récit, Die brennende Wand, respire l'exiguïté, contrairement à celui de Regina qui, elle, souffrait de l'espace vide dans sa maison. C'est à la suite de la chute de l'ancienne RDA qu'Henry, autrefois vacher, s'est vu amené à se reconvertir et a pu trouver refuge dans une troupe de cascadeurs qui parcourt villes et villages où il sert d'homme à tout faire. Il vit ainsi à l'étroit dans un réduit situé derrière la cabine du chauffeur, sans confort, sans lumière naturelle, en possession du strict minimum. Il a troqué par nécessité la vie à la campagne pour un paysage urbain de conteneurs, de rails, de snack-bars et de barres d'immeubles.

9 On a dit des personnages de Peter Stamm qu'ils étaient en attente de quelque chose susceptible de changer leur vie - une attitude que l'auteur ressent comme représentative de son époque ${ }^{8}$. Henry, lui aussi, est dans l'expectative. Sa vie de vagabondage n'a pas occulté le désir de fonder une famille, de connaître le même bonheur que celui que semblent goûter ses collègues. Or, sa vie d'errance rend toute relation amoureuse difficile. D'un côté, Henry croit avoir vu sa vie s'améliorer depuis la chute du Mur - il semble même considérer les voyages qu'il entreprend comme une aubaine. D'un autre côté, il souffre de sa solitude, comme de nombreux personnages de Stamm qui semblent «très seuls et apatrides 9 . " Manuela, la serveuse chez Mac Donalds qu'il emmène dans son réduit, son « clapier » (27), se rend vite compte qu'il est souvent seul et qu'il a peu l'habitude de rencontres érotiques. Dans ce récit, Peter Stamm met en évidence que le temps presse pour Manuela et Henry, car le départ de la troupe est imminent. Il faudrait à Manuela une détermination qu'elle peut difficilement avoir pour suivre Henry qu'elle connaît à peine. Certes, elle ne peut imaginer vivre avec Henry dans ce réduit, sans baignoire, n'arrive pas à se représenter comment ses futurs enfants pourraient allier vie d'errance et suivi scolaire, pourtant elle se projette aussi dans l'avenir, comme nous le montre la scène où elle s'imagine étendre du linge devant une caravane. Pour elle, comme pour Henry, cette brève rencontre n'a pas été sans conséquence. Henry ôte du mur l'affiche érotique qui le couvrait pour ne pas blesser la sensibilité de Manuela qui peut-être reviendra, il fait le lit pour qu'elle s'y sente plus à l'aise et défonce le mur de flamme en voiture pour l'amour de Manuela. Manuela, quant à elle, n'est pas restée insensible à Henry et est heureuse de leur rencontre. De minces détails nous le montrent : le suçon qu'elle arbore avec fierté devant son amie Denise et sa certitude de ne pas avoir été la victime d'un beau parleur. Cette histoire apparemment peu spectaculaire, comme souvent chez Peter Stamm, cache une situation tragique. Alors qu'Henry croit pouvoir impressionner Manuela et l'emmener après la représentation à nouveau dans son réduit, il paie de sa vie une cascade qui tourne mal. Manuela, quant à elle, s'étonne de l'absence d'Henry, croit fermement qu'il a dit vrai mais ne cherchera pas à s'enquérir de sa disparition, car l'incertitude la gagne alors devant une relation qui semble vouloir rester éphémère: "Ça ne fait rien, dit Manuela. C'était pas important. Ça n'aurait de toute façon rien donné » $(39)^{10}$. Elle n'apprendra pas la mort d'Henry. 
10 Les histoires de Peter Stamm mettent souvent en scène des situations de la vie courante, des événements que l'on a qualifiés de peu frappants. Cela s'applique aussi au troisième récit qui donne au recueil son titre, In fremden Gärten. Une femme dont le nom reste inconnu est chargée de s'occuper de l'entretien des plantes en l'absence de Ruth, sa voisine. La raison du départ de Ruth n'est révélée qu'à la fin du récit et le lecteur pense spontanément à un voyage d'agrément. Le lieu dans lequel pénètre la voisine semble figé ; persiennes closes, objets en attente dans la cuisine, téléphone qui sonne en vain et résonne dans ce lieu marqué par l'absence. Les gestes de la voisine de Ruth sont mesurés, comme empreints d'une déférence que l'on n'aurait peut-être pas pour quelqu'un parti seulement en voyage. La voisine semble pénétrer dans ce lieu étranger pour elle comme dans un lieu saint, avec un respect excessif. Elle hésite par exemple avant d'ouvrir les portes, trie consciencieusement le courrier, n'ose pas toucher au verre rempli d'eau dans la cuisine. Elle va être cependant l'auteur d'une seule indiscrétion, non par curiosité vraisemblablement mais par compassion, comme le fait penser l'issue du récit. La carte d'Anniversaire de Ruth que la voisine prend en main et lit en son absence sert à déclencher le récit des grandes lignes de la vie de Ruth. On apprend que Ruth et sa famille semblent attirer le malheur. Il est question d'une mère indigente vivant retirée, d'un père ayant passé sa vie dans une boutique sombre et peu fréquentée et qui s'est finalement suicidé, de frères vandales dont l'un, drogué, est mort. Puis du mariage apparemment heureux de Ruth qui pourtant a échoué. La dernière page nous apprend ainsi que Ruth n'a pas quitté les lieux pour un voyage d'agrément mais pour un séjour en clinique, assurément la suite logique de la séparation de son mari qui, pour la première fois, l'a brisée. Le pense-bête que la voisine trouve dans la maison de Ruth et qui contient les objets que cette dernière voulait emporter en clinique émeut tant la voisine qu'elle envisage de rendre visite à celle qu'elle ne connaissait que de voisinage. Ce lieu étranger dont elle avait la garde lui a rendu Ruth plus proche, plus familière.

11 Die ganze Nacht, récit de quelques pages seulement, nous montre comment, à la suite d'une tempête de neige, les lieux et le personnage central sont transformés. Le récit se déroule, comme souvent chez Stamm, à l'étranger ; il choisit ici la ville de New York. Le personnage masculin dont on ignore le nom attend le retour de son amie dont le vol a été détourné sur Boston en raison des intempéries. La crise entre eux pourrait surgir à son retour, semble-t-il, car le personnage central a décidé de mettre certaines choses au clair avec son amie, des choses que le lecteur ne connaît pas. Mais l'impact de la neige donne un caractère solennel aux lieux qu'il connaît habituellement sous d'autres traits, fait naître une sensation de douceur qui va changer le sentiment du personnage masculin. «L'ambiance était solennelle, personne ne parlait, c'était comme s'ils étaient tous témoins d'un miracle » (59) ${ }^{11}$ dit-on des clients du bar où le personnage central se rend et qui, en raison de la neige, restera exceptionnellement ouvert toute la nuit. La neige rend plus aléatoires les différends et fait oublier au personnage principal les phrases qu'il s'était répétées et dont il croyait avoir besoin. Le lieu qu'il semblait connaître et qui sous la neige dégage une toute autre atmosphère, plus douce, plus feutrée, le pousse à laisser de côté toute explication et à se laisser aller à la fin du texte à sa joie des retrouvailles.

12 Éric, le personnage de Wie ein Kind, wie ein Engel connaît lui une vie d'errance due à ses obligations professionnelles - des voyages qui occupent les deux tiers de son temps. Toujours sur les routes en Amérique du Nord et en Europe, il a à présent une vision 
moins positive de ses déplacements qui, au début de sa carrière, ont constitué l'attrait essentiel du poste qu'il occupe à la révision des comptes dans une multinationale du secteur alimentaire : «Il aimait ça, courir le monde et rencontrer des gens » $(64)^{12}$. Mais ce sentiment de satisfaction a laissé place à la routine et à présent, ses déplacements lui sont devenus un fardeau. Alors qu'il vit grâce à ces voyages une vie cossue dans de bons hôtels, tous frais payés, les pays étrangers ne lui semblent plus attrayants et l'impression de déjà-vu le hante: "Les chambres d'hôtel se ressemblaient, les restaurants se ressemblaient, comme les conversations avec les collègues, les aéroports, les villes $\gg(65)^{13}$.

13 Après une présentation générale de la vie professionnelle d'Éric, Peter Stamm s'attarde sur un cas particulier, sur la relation qu'entretient Éric avec un certain Valdis dans un pays de l'Europe de l'Est, en Lettonie et il nous montre, dans cette vie ponctuée par les voyages, la difficulté d'établir des liens autres que fugitifs. Une lettre et un appel au secours auraient pu faire d'Éric et de Valdis des amis de toujours, mais le lien qui les unit est trop ténu - ils ne se voient qu'une fois par an -, la demande de Valdis surdimensionnée, ce qui empêchera Éric d'aider Valdis. Celui-ci, en effet, s'adresse par écrit à Éric en lui demandant de l'aider financièrement, sa femme étant atteinte d'un cancer nécessitant un traitement au coût exorbitant de 100000 francs suisses. Cette demande démesurée, fruit d'une situation désespérée, Valdis la confie à une relation de passage parce que pour lui des liens se sont tissés et qu'il sait qu'Éric, vivant en Suisse, dispose d'une plus grande autonomie financière que lui. C'est à cet homme qu'il ne voit qu'une fois par an que Valdis confie ce qu'il a de plus cher, la vie de sa femme. C'est tout d'abord l'incompréhension et la gêne qui dominent chez Éric et il ne saura pas maitriser cette situation mais prendra le parti d'ignorer la demande de Valdis, malgré la promesse faite dans son message électronique. Au lieu d'aborder avec Valdis cette question épineuse dont dépend la vie d'une personne, de prendre position, de ne pas laisser s'installer l'indifférence, Éric fuit devant la responsabilité, tandis que Valdis tombe dans un mutisme, par gêne vraisemblablement. Il y a une succession de situations ratées et de non-dits. L'amitié que lui atteste Valdis un soir, à moitié ivre, a un goût d'amertume et de désespoir. Les voyages qu'entreprend Éric dans le pays de Valdis n'ont pu faire naitre que des relations humaines superficielles. Devant la souffrance de Valdis qu'il peut supposer, Éric se défile et rentre en Suisse avec certes un «étrange désarroi » qui cependant s'estompe, comme nous le fait penser le début du texte.

Pour son récit suivant, Fado, Peter Stamm choisit la ville de Lisbonne - il s'agit cette fois d'une brève rencontre entre deux Canadiennes, Rachel et Antonia, un Portugais Luis et le narrateur. Mais de cette ville, nous ne voyons presque rien, un bar à Fado, une discothèque et une chambre dans une pension. Le désir de fuir l'Europe a amené le narrateur jusqu'à Lisbonne où il désire s'embarquer et laisser derrière lui le continent européen. Dans son désir d'aller plus loin, le narrateur considère le voyage hors d'Europe comme une promesse de bonheur. Cependant la chanson du chanteur de Fado semble s'appliquer à sa situation: "Quand tu ne sais pas où tu vas, pourquoi ne t'arrêtes-tu pas de marcher?» (85) ${ }^{14}$. Ainsi, comme Éric dans Wie ein Kind, wie ein Engel, la ville étrangère dans laquelle il se trouve ne le captive pas. Il reste en marge des autres, ne prend pas le temps de visiter les lieux, se satisfait par contre d'une rencontre fortuite avec deux Canadiennes, marquée par la banalité. La relation qu'il noue avec elles est une tentative de rompre avec le sentiment de solitude qui l'habite et il est « ravi d'être avec des gens » (87). Mais la fin nous montre que les liens qui les unissent 
sont, là aussi, ténus, sans avenir. Après avoir passé une partie de la nuit avec les deux femmes, il juge bon de jeter l'adresse qu'elles lui ont donnée, montrant ainsi le peu de valeur qu'il accorde à la relation qu'il vient de nouer. Des connaissances, qui, dans cette vie d'errance, semblent se faire et se défaire sans souffrance, dans une indifférence générale. La dernière scène qui a lieu dans une église de Lisbonne au moment de l'office nous montre le narrateur en marge de cette communauté. Tandis que chacun tend à son voisin une main amicale, le narrateur, qui a tenu à s'asseoir au dernier rang, ne reçoit aucune poignée de main fraternelle. Il semble condamné à vivre dans l'errance et la solitude et à se satisfaire de rencontres fortuites auxquelles manquent la profondeur.

On a comparé le personnage du récit suivant, de Alles, was fehlt, David, à L'Étranger de Camus, par son détachement dans la vie et son désintérêt. Comme Henry dans Die brennende Wand parcourt par obligation professionnelle les routes et Éric dans Wie ein Kind, wie ein Engel change sans cesse de lieu, David que nous voyons à son arrivée à l'aéroport de Londres doit séjourner dans la capitale britannique pendant un an au moins pour des raisons professionnelles. Le premier paysage que nous entrevoyons est celui des gratte-ciel des Docklands qui laisse David sans émotion. David ne se réjouit pas d'avoir quitté la Suisse pour l'Angleterre. S'il l'a fait, c'est parce qu'on a exigé de lui une «flexibilité géographique » et il affirme laconiquement: "je n'ai aucune envie d'être ici» (103) $)^{15}$. Le paysage qui l'environne dans la tour qu'il habite est un paysage urbain ; on y voit les gratte-ciel de Canary Wharf, la Tamise, un parc municipal et une aire de jeux. Dans les 151 appartements presque identiques qui forment le complexe où loge David, l'anonymat règne et le confort semble avoir remplacé les contacts humains. L'appartement de fonction qu'il occupe lui procure en effet toutes les commodités cuisine de designer, téléviseur géant sur roulettes, robinetterie de marque etc. - mais sa solitude est évidente. Cependant, il est peu liant, comme nous le montre son refus d'être accompagné par Rosemary, la secrétaire, jusqu'à Greenwich et son saisissement lorsqu'il aperçoit dans ce monde dominé par le béton, la présence de sa voisine japonaise à qui il n'ose adresser la parole.

On a affirmé des histoires de Peter Stamm qu'elles étaient peu spectaculaires. Cela vaut pour Alles, was fehlt. Il ne se passe presque rien dans ce récit où domine, à côté du sentiment de solitude, la nostalgie des contacts humains dans un lieu étranger. David tente dès son arrivée de se persuader que Londres est une ville attrayante, que le sentiment qu'il a de se sentir étranger est injustifié, mais sans véritablement y croire : «Il allait sortir, aller au concert, au cinéma, voir des musicals. Il ferait des connaissances » $(104)^{16}$. Comme Éric dans Wie ein Kind, wie ein Engel était blasé par ses déplacements et ne voyait rien ou presque des villes étrangères dans lesquelles il séjournait, comme le narrateur de Fado ne prenait aucun plaisir à visiter Lisbonne, décidé qu'il était à tourner le dos à l'Europe, David, contraint de vivre à l'étranger pour garder son emploi, ne semble savoir profiter de ces lieux étrangers qui pourtant pourraient l'ouvrir sur une autre culture. La nouvelle se termine cependant sur un court dialogue avec la voisine japonaise à qui, en toute simplicité, il dit bonjour, donnant ainsi l'espoir de nouer des liens dans cette cité étrangère. Mais leur dialogue demeure très bref et le lecteur reste dans l'interrogation.

Le très court récit Der Aufenthalt s'ouvre sur un hall de gare où des jeunes gens, assis sur des sacs de voyage, attendent, par une chaleur accablante, la venue d'un train. La phrase que prononce Daniel est symptomatique des personnages de Peter Stamm. « On est toujours en route pour quelque part » dit-il. Pour ajouter cependant: "Même les 
malades. Même les morts » $(121)^{17}$. Un train a en effet attiré l'attention des jeunes gens, un train de la Croix-Rouge en route pour Lourdes et à bord de ce train, un pèlerin est décédé. Une vague sensation de malaise et d'effroi devant la maladie s'empare des jeunes gens, irrités en outre par les regards scrutateurs des malades qui, aux fenêtres, semblent attendre eux aussi une mort prochaine. Dans une interview pour le Tageszeitung, Peter Stamm en dit plus sur l'origine de ce bref récit :

Oui, cela peut illustrer la vie, le fait d'être toujours en chemin. J'ai travaillé durant des années à l'aéroport et ai chargé et déchargé des avions. Il m'est arrivé plusieurs fois de charger des cercueils. On envoie des cadavres par exemple en Turquie, ce qui a quelque chose d'absurde. C'est pourquoi je connais le fait de faire voyager des morts ${ }^{18}$

Si Peter Stamm a l'habitude de mettre en scène de jeunes personnages, il projette ici, comme dans Der Besuch, son regard sur la fin de vie, nous montrant à la fin du récit les jeunes gens pris de compassion à la nouvelle de la mort du pèlerin, comprenant après coup la douleur et la nervosité des voyageurs en route pour Lourdes.

L'histoire suivante, celle du Dr. Kennedy et de ses filles, Deep Furrows, part, elle, d'un fait autobiographique, comme l'explique Peter Stamm dans une interview pour Büchergilde. L'auteur dit avoir rencontré en 1982 une personne portant le même nom que le Dr. Kennedy. Celui-ci lui aurait raconté avoir épousé, en tant qu'Irlandais, une Allemande pour importer du sang neuf dans son pays. Le reste est le fruit de son invention, confie Peter Stamm, qui, à l'époque, a préféré ne pas donner suite à l'invitation du docteur qu'il trouvait trop bizarre.

Comme dans de nombreux textes de Peter Stamm, le narrateur est un jeune voyageur. Dans un pub irlandais, celui-ci fait la connaissance d'un orthopédiste, le Dr. Kennedy, qui se montre dès le début intéressé par cet étranger car il a pour but de marier ses filles et s'enchante de l'apport de sang neuf que pourrait constituer le mariage du jeune narrateur avec l'une d'elles. On apprend par le docteur la propension des Irlandais à privilégier les mariages consanguins d'où le Dr. Kennedy voit découler tous les maux sociaux du pays où il vit, désordre, chômage, fanatisme religieux et alcoolisme. Pour le Dr. Kennedy, la Suisse est un pays béni, parce que les peuples se sont mêlés. Lui-même prône l'ouverture mais vit retranché dans un bungalow quelque peu délabré. Ses filles sont elles aussi attachées à ce lieu familier - Gwen souhaiterait par exemple passer sa vie dans le jardin qu'elle entretient avec amour, parmi la beauté des fleurs et attend la confirmation du narrateur lorsqu'elle lui demande : « N'est-ce pas merveilleux ? [...] La musique, la maison, tout?» (133) ${ }^{19}$ À l'opposé du narrateur qui vite va ressentir le besoin de quitter les lieux pour échapper à la sédentarité et aux espoirs du Dr. Kennedy, Gwen s'invente une histoire où de prétendus grands-parents qu'elle semble vénérer ont fait preuve d'un attachement sans faille à leur demeure en étant enterrés là où ils ont vécu. C'est un sentiment de soulagement qui s'empare du narrateur, lorsqu'il quitte ce lieu et ses propriétaires, certes très prévenants mais trop prenants. Il est évident que les liens tissés ici resteront une fois de plus sans conséquence et cette relation amorcée ne sera pas poursuivie.

Dans Der Kuß, le récit qui clôture In fremden Gärten, Peter Stamm met face à face un père d'un certain âge et sa fille. Celle-ci a absolument tenu à quitter le Danemark, son pays d'origine, pour s'installer en Suisse. Cette décision est la pierre d'achoppement dans la relation des deux personnages. Par leur âge, leur éducation, leur goût, père et fille ont une appréhension toute différente des voyages. Le voyage d'Odense en Suisse est pour le père le premier voyage qu'il effectue seul. Il est ainsi mal à l'aise. Jeune, il n'avait pas 
d'argent pour voyager et a fondé tôt une famille : «Jadis, on restait où on était » $(157)^{20}$; c'est ainsi qu'il explique son propre choix. Les quelques voyages qui l'ont conduit, lui et sa famille, en Italie ou en Espagne ont cessé à la mort de sa femme. Inger, sa fille, par contre a toujours porté en elle le désir de quitter son pays natal: "Chaque jour elle voyait, elle entendait les trains qui partaient vers le Sud» (159) ${ }^{21}$. C'est un plus grand anonymat, synonyme pour elle de liberté, qu'elle voulait trouver à l'étranger, considérant une vie en famille comme un recul: «Elle, elle voulait avancer, pas reculer " (159) ${ }^{22}$. Elle vit ainsi sans attache en tant que serveuse - une décision que son père conteste, car grâce au métier qu'elle a appris au Danemark, son statut social pourrait être bien meilleur. La relation qui unit les protagonistes est caractérisée lors des retrouvailles par la gêne, la distance et l'incompréhension, même si l'amour n'est pas absent. Père et fille vont devoir tous deux vivre en promiscuité dans la petite chambre qu'occupe Inger. Ce lieu exigu va favoriser leur rapprochement. Une randonnée va par ailleurs montrer au père qui n'a pas l'habitude de la montagne, après quelques moments de malaise, la joie de découvrir d'autres horizons. Le récit s'achève sur le désir du père de satisfaire sa fille et avec elle d'aller plus au Sud - une direction qui déjà toute jeune attirait sa fille avec force.

Une grande partie des personnages du recueil In fremden Gärten sont des voyageurs pour qui la connaissance de nouvelles contrées n'apporte pas ou plus l'épanouissement escompté. Ils n'ont pas l'opportunité et l'envie d'apprivoiser ces pays étrangers qui les laissent insensibles. Ils vivent pour la plupart en marge des autres, condamnés à poursuivre leur vie d'errance et à se satisfaire de relations de passage. Pour Peter Stamm cependant, planter un décor dans une ville étrangère à la Suisse est un besoin "Il m'est plus facile d'écrire sur des lieux qui me sont étrangers " ${ }^{23}$, confie-t-il, une habitude qu'il reconnaît dans la mentalité suisse, cosmopolite et attirée par les voyages ${ }^{24}$. Mais la description des paysages étrangers dans In fremden Gärten reste schématique. On ne reconnaît qu'en marge ces lieux étrangers et le terme de " paysages aléatoires » que l'auteur a donné à son deuxième roman convient aux coulisses de ses récits.

On a dit des histoires de Peter Stamm qu'elles étaient sans happy end. L'auteur a luimême conclu que la vie, scellée par la mort, était elle-même sans happy end et a vu ses histoires à son image. Cependant à côté des déshérités de la vie comme Henry dans Die brennende Wand, il y a dans ce nouveau recueil ceux qui font une expérience positive, comme Regina dans Der Besuch, la voisine dans In fremden Gärten, le personnage masculin dans Die ganze Nacht ou bien le père dans Der Kuß. Ainsi, à côté des solitaires, il y a ceux qui parviennent à aller au devant des autres ; Regina montre de la compréhension pour la relation de sa petite-fille malgré ses propres frustrations, la voisine de Ruth de la compassion pour cette dernière et le père d'Inger réussit à la fin à abandonner ses a priori pour s'ouvrir aux souhaits de sa fille et donner une nouvelle impulsion à leur relation. Il y a donc des moments de bonheur dans ce nouveau recueil - même s'ils restent furtifs.

Quoique les textes de Peter Stamm semblent être au premier regard non politiques, ils contiennent une réflexion sociale sous-jacente inspirée de ses séjours à l'étranger. Dans 
son nouveau recueil In fremden Gärten, Peter Stamm ne considère pas qu'il ait fait abstraction de thèmes politiques, comme il le confie dans une interview :

Non, je pense tout simplement qu'il est difficile de faire de la politique en

littérature. Mais il s'agit aussi dans cet ouvrage de questions politiques ${ }^{25}$

Des questions politiques qui font surface de façon souvent inattendue comme lorsque Luis dans Fado raconte qu'il vient de Faro, du Sud du Portugal, mais qu'il n'y a pas de travail là-bas, ou lorsque le Dr. Kennedy évoque les comités d'autodéfense dans les quartiers pauvres d'Irlande. Ses séjours à l'étranger ont montré à Peter Stamm notamment que le bien-être n'était pas une évidence pour tous, comme nous le fait penser l'appel au secours de Valdis dans Wie ein Kind, wie ein Engel. À la question que lui pose un journaliste, à savoir ce qu'il a retenu de ses voyages à l'étranger et quel enseignement il en tire pour son propre pays, Peter Stamm répond :

Oui, le fait que notre bien-être ne va pas de soi. Qu'il est là aux dépens d'autrui. Que l'on peut avoir dans la vie d'autres priorités que celles que nous avons. Mais j'ai appris aussi à apprécier de nombreuses choses en Suisse. Le fait de ne pas se compliquer la vie, l'exactitude, le cosmopolitisme et la grande éthique de travail des Suisses. Le fait qu'ils s'identifient aussi avec leur pays dont ils se sentent responsables ${ }^{26}$.

\section{NOTES}

1. www.peterstamm.ch. Warum ich schreibe.

2. www.culturactif.ch.

3. www.peterstamm.ch. Theater.

4. « Ein bisschen auch von der Schweizerischen » www.woxx.lu.

5. «Nomaden, die durch die Weltgeschichte treiben» (www.dradio.de/dlf/sendungen/ buechermarkt/165941.)

6. «Meine Nationalität war für mich nie besonders wichtig. Ich habe immer versucht, mich an das Land anzupassen, in dem ich gerade war. Sonst hätte ich ja genauso gut in der Schweiz bleiben können » (www.woxx.lu/text_show.php?textid=855).

7. P. Stamm: D'étranges jardins. Traduit de l'allemand par Nicole Roethel. Christian Bourgois éditeur, 2003, p.17. Les numéros de page de la traduction française seront placés entre parenthèses après la citation. "Seit langer Zeit hatte sie wieder das Gefühl, das Haus sei voll » (P. Stamm: In fremden Gärten. Arche, p. 16).

8. «Aber das ist doch ein bisschen die Situation im Moment, dass alle auf etwas warten » (Büchergilde: Interview mit Peter Stamm. www.buechergilde.de/thema/archiv/ exklusivinterviews/stamm-teil1.shtml).

9. Ib.

10. «"Ist ja egal”, sagte Manuela, "War ja nichts. Wäre ja sowieso nichts geworden” » (P. Stamm: In fremden Gärten, p. 35).

11. «Die Stimmung war festlich, niemand sprach, es war, als seien sie alle Zeugen eines Wunders » (Id. p. 51).

12. «Er mochte es, herumzukommen und Leute kennenzulernen » (Id. p. 56). 
13. «Die Hotelzimmer glichen sich, die Restaurants glichen sich, die Gespräche mit den Kollegen, die Flughäfen, die Städte » (Id., p. 57).

14. « Wenn du nicht weißt, wohin du gehst, warum hörst du nicht auf zu laufen » (Id. p. 75).

15. «Ich will hier nicht sein » (Id., p. 91).

16. « Er würde ausgehen, in Konzerte, ins Kino, in Musicals. Er würde Leute kennenlernen » (Id. p. 92).

17. «"Alle sind immer irgendwohin unterwegs", sagte Daniel, "sogar die Kranken. Sogar die Toten" » (Id. p. 108).

18. « Ja, das kann ein Bild fürs Leben sein, das Unterwegs-Sein. Ich habe jahrelang am Flughafen gearbeitet und Flugzeuge be- und entladen. Da kam es immer wieder vor, dass wir Särge verladen haben. Man schickt Leichen zum Beispiel in die Türkei, was letztlich etwas Absurdes hat. Daher kenne ich die Situation, in der Tote verschickt werden » (www.buechergilde.de/thema/archiv/ exklusivinterviews/stamm_teil2.shtml).

19. "“Ist es nicht wunderschön?" sagte Gwen. "Die Musik, das Haus und alles ?” » (P. Stamm: In fremden Gärten, p. 119).

20. «Damals blieb man, wo man war » (Id. p. 140).

21. «Jeden Tag sah sie die Züge, hörte sie die Züge, die nach Süden fuhren » (Id. p. 142).

22. « Sie wollte weiter, nicht zurück» (Id. p. 143).

23. «Es fällt mir leichter über Orte $\mathrm{zu}$ schreiben, die mir fremd sind» www.woxx.lu/ text_show.php?textid=855

24. « die Schweizer sind sehr weltoffen und reisefreudig » (Ib.)

25. « Nein. Ich denke einfach, in der Literatur ist es sehr schwierig, politisch zu sein. Aber es geht auch in diesem Buch letztlich um politische Fragen" www.buechergilde.de/thema/archiv/ exklusivinterviews/stamm_teil2.shtml.

26. «Ja, dass unser Wohlstand nicht selbstverständlich ist. Dass er teilweise auf Kosten anderer geht. Dass man auch andere Prioritäten im Leben haben kann, als wir sie haben. [...] Aber ich habe auch vieles an der Schweiz schätzen gelernt. Das Unkomplizierte, die Sorgfalt, die Weltoffenheit und die hohe Arbeitsmoral der Schweizer. Und ihre Identifikation mit dem Land, für das sie sich verantwortlich fühlen. » www.woxx.lu/text_show.php?textid=855.

\section{RÉSUMÉS}

Depuis la parution de son roman Agnes (1998), Peter Stamm fait partie des jeunes espoirs de la littérature suisse contemporaine. En 2003 a paru au Arche Verlag un nouveau volume de récits In fremden Gärten dont le titre est inspiré de Goethe. Comme souvent chez Peter Stamm, les histoires qu'il écrit ont pour coulisse des pays étrangers. Les personnages qui évoluent dans ce nouveau recueil sont pour la plupart solitaires, certains vivant par nécessité ou par choix loin de leur pays d'origine. De ces lieux étrangers, il ne semblent pas toujours profiter. Ils sont souvent en attente d'un événement qui pourrait donner une nouvelle impulsion à leur vie et qui pour certains n'arrive pas. À côté des personnages solitaires, il y a ceux qui s'ouvrent aux autres et font une expérience positive, réconciliation dans $\mathrm{Der} \mathrm{Ku} \beta$, compassion dans In fremden Gärten ou compréhension dans Der Besuch.

Seit dem Erscheinen des Romans Agnes (1998) zählt Peter Stamm zu den neuen Hoffnungen der zeitgenössischen Schweizer Literatur. 2003 ist im Arche Verlag ein neuer Erzählband erschienen, 
In fremden Gärten. Auch in diesem Band ist es für Peter Stamm typisch, daß seine Handlungsorte selten in der Schweiz sind. Seine Geschichten spielen in New York, Lettland, England oder Italien. Der Autor gibt selber zu, leichter über Orte zu schreiben, die ihm fremd sind. Man hat seine Figuren einsame Nomaden genannt, die durch die Welt treiben. Auch dies gilt für diesen neuen Erzählband. Diese Figuren sind oft auf der Suche nach einer Veränderung in ihrem Leben und profitieren wenig von ihrem Reiseleben. Neben den Geschichten, die ohne happy end an fremden Orten spielen, sind welche, die eine positive Erfahrung der Figuren verzeichen, Versöhnung in Der Kuß, Mitgefühl in In fremden Gärten oder Verständnis in Der Besuch.

INDEX

oeuvrecitee In fremden Gärten, Agnes, Der Kuß, Der Besuch

\section{AUTEURS}

\section{JOËLLE STOUPY}

Université du Littoral-Côte d'Opale (Boulogne-sur-Mer) 